\title{
Axitinib for Gemcitabine-refractory Advanced Biliary Tract Cancer: Report of 5 Cases
}

\author{
NAOHIRO OKANO, AKIYOSHI KASUGA, KIRIO KAWAI, TAKAAKI KOBAYASHI, \\ DAISUKE NARUGE, FUMIO NAGASHIMA and JUNJI FURUSE
}

Department of Medical Oncology, Kyorin University Faculty of Medicine, Tokyo, Japan

\begin{abstract}
Background/Aim: Vascular endothelial growth factor receptor (VEGFR) has been identified as a treatment target for biliary tract cancer (BTC) and axitinib is a selective inhibitor of vascular endothelial growth factor receptor (VEGFR)-1/2/3. This study was conducted as a preliminary evaluation of the safety and efficacy of axitinib for patients with advanced BTC. Patients and Methods: Patients refractory to gemcitabine-based regimens were administered axitinib at the dose of $5 \mathrm{mg}$ twice daily. Results: Five patients ( 3 male and 2 female) with a median age of 68 years were enrolled. Although 3 patients developed treatment-related grade 3/4 adverse events (AEs), none of these patients required discontinuation of the protocol treatment due to the AEs. Partial response (PR) was achieved in 1 patient, with a $67 \%$ reduction. The response was classified as stable disease (SD) in 3 patients and as progressive disease $(P D)$ in 1 patient. Overall survival (OS) and progression-free survival (PFS) ranged from 2.0 to 19.9 months and 1.5 to 7.4 months, respectively. Conclusion: This preliminary study suggested that axitinib is well-tolerated and might exert promising activity in patients with BTC.
\end{abstract}

Biliary tract cancer (BTC) consists of extrahepatic cholangiocarcinoma (ECC), gallbladder cancer (GBC) and ampulla of Vater cancer (AVC); intrahepatic cholangiocarcinoma (ICC) is also included in clinical trials of chemotherapy. The incidence of BTC is especially high in Asia and Latin America and approximately 25,000 patients are annually diagnosed as having BTC in Japan (1). Most BTC patients are diagnosed at an advanced stage of the

Correspondence to: Naohiro Okano, MD, Department of Medical Oncology, Kyorin University Faculty of Medicine, 6-20-2, Shinkawa, Mitaka-shi, Tokyo, 181-8611, Japan. Tel: +81 422475511, Fax: +81 422440729, e-mail: naohiro-okano@ks.kyorin-u.ac.jp

Key Words: Biliary tract cancer, chemotherapy, axitinib, gemcitabine, vascular endothelial growth factor. disease because of absence of symptoms in the early stage. Furthermore, even after radical resection, most patients develop recurrence. Thus, systemic chemotherapy plays an important role in the treatment of BTC and, based on the results of two randomized control trials in which gemcitabine (GEM) plus cisplatin was compared with GEM alone $(2,3)$, GEM plus cisplatin is currently recognized as the global standard chemotherapy for patients with advanced BTC.

While GEM plus cisplatin is applied as first-line chemotherapy, most patients show tumor progression over time; no effective second-line treatment has been established for BTC. To date, some single-arm trials have shown modest efficacy of second-line chemotherapy with a median overall survival (OS) and progression-free survival (PFS) of 4.1 to 13.5 months and 1.6 to 5.4 months, respectively (4-13). Thus, establishment of second-line chemotherapy for patients with advanced BTC is urgently needed.

Overexpression of vascular endothelial growth factor (VEGF) is identified as one of the important poor prognostic factors in patients with various solid cancers, including BTC $(14,15)$. Therefore, VEGF receptor (VEGFR) has attracted attention as a treatment target in patients with advanced BTC. Axitinib is an orally administered selective inhibitor of VEGFR-1/2/3. A preclinical examination using xenografts demonstrated the anti-tumor activity of axitinib against cholangiocarcinoma (16). However, to date, the safety and efficacy of axitinib has not been examined in patients with BTC. Therefore, this study was conducted to achieve a preliminary evaluation of the safety and efficacy of axitinib in patients with BTC and, thereby, assess whether it might be worthwhile conducting further clinical trials of axitinib for BTC patients.

\section{Patients and Methods}

Patients' eligibility. The eligibility criteria for enrollment in this study were: patients who had been diagnosed as having recurrent or unresectable BTC, including ICC, ECC, GBC or AVC; had histologically confirmed adenocarcinoma; were 20 years of age or older; had an Eastern Cooperative Oncology Group (ECOG) 
Performance Status (PS) of 0 or 1; showed progressive disease under GEM-based chemotherapy or after discontinuation of GEM-based chemotherapy due to the emergence of adverse events (AEs); had $\geq 1$ measurable lesion; had no evidence of brain metastasis; had no evidence of massive ascites and/or pleural effusion; had no evidence of preexisting uncontrolled hypertension; had preserved organ functions (white blood cell count $\geq 3,000 / \mathrm{mm}^{3}$, neutrophil count $\geq 1,500 / \mathrm{mm}^{3}$, hemoglobin level $\geq 8.0 \mathrm{~g} / \mathrm{dl}$, platelet count $\geq 100,000 / \mathrm{mm}^{3}$, serum creatinine level $\leq 1.5 \mathrm{mg} / \mathrm{dl}$, serum total bilirubin level $\leq 2 \mathrm{mg} / \mathrm{dl}(\leq 3 \mathrm{mg} / \mathrm{dl}$ in patients with biliary drainage) and serum aspartate transaminase (AST) and alanine transaminase (ALT) levels $\leq 75$ IU/l ( $\leq 150 \mathrm{IU} / 1$ in patients with biliary drainage)); urinary protein level $\leq 1+$ by the dipstick test or $<2,000 \mathrm{mg} /$ day; had a life expectancy of $\geq 3$ months; were willing to provide written informed consent.

The exclusion criteria were: double cancer; active hemorrhagic ulcer or active diverticulitis; surgery within 28 days prior to enrollment in the study; radiation therapy within 14 days prior to enrollment in the study; serious gastrointestinal disorder; serious complications, such as cardiac disease, renal disease, uncontrolled diabetes mellitus; active infections; active epilepsy; pregnant or lactating women. This study was conducted with the approval of the local Institutional Review Board.

Treatment methods. Eligible patients were administered axitinib (kindly provided by Pfizer Japan Inc., Shibuya-ku, Tokyo, Japan) at a starting dose of $5 \mathrm{mg}$ orally twice daily. The treatment was continued until disease progression, emergence of intolerable AEs or patient refusal to continue the treatment.

Two dose-level modifications for toxicity (to $3 \mathrm{mg}$ or $2 \mathrm{mg}$ orally twice daily) were allowed. In patients able to tolerate the dose of 5 $\mathrm{mg}$ orally twice daily, dose escalation (to 7 or $10 \mathrm{mg}$ orally twice daily) was also permitted.

Assessment of the response and toxicity. Complete hematological tests, blood biochemistry tests, urine tests and physical examination, including blood pressure measurement, were carried out at least every 2 weeks. In addition, measurement of thyroid hormone levels was carried out every 4 weeks until two months after the initiation of treatment and every 6 weeks thereafter.

Computed tomography (CT) or magnetic resonance imaging (MRI) was performed every 6 weeks for evaluation of the treatment efficacy, with the tumor response being evaluated in accordance with the Response Evaluation Criteria in Solid Tumors 1.1. AEs were recorded in accordance with the Common Terminology Criteria for Adverse Events 4.0.

Study design. This study was an open-label, single-center study. The objective was to confirm the feasibility of using axitinib for GEMrefractory BTC patients. The standard dose of axitinib of $5 \mathrm{mg}$ twice daily was evaluated in this study. The sample size was set at 3 to 6 patients, to evaluate the dose-limiting toxicities (DLTs). DLT was defined as follows: (i) Grade 4 neutropenia persisting for more than 7 days; (ii) Grade 4 thrombocytopenia; (iii) Grade 3 or more severe non-hematological toxicity not controlled by appropriate supportive cares; (iv) uncontrolled hemoptysis; (v) urinary protein excretion more than $3.5 \mathrm{~g} / 24$ hours; (vi) less than $75 \%$ of the dose of axitinib in the first cycle due to toxicities.

\section{Results}

Patients' characteristics. Five patients ( 3 males and 2 females) were enrolled in this study between May 2013 and March 2015. The patients' characteristics are shown in Table I. The median age was 68 years (range=34-77). Four patients had an ECOG PS of 0. The tumors were diagnosed as ICC, ECC and GBC in 2, 2 and 1 patients, respectively. Three patients had metastatic disease, and 2 patients had recurrent disease. Before the start of axitinib treatment, 2 patients had received one treatment regimen and 3 patients had received two treatment regimens. The previous treatments included GEM plus cisplatin in 4 patients, S-1 monotherapy in 2 patients, GEM plus $\mathrm{S}-1$ in 1 patient and GEM monotherapy in 1 patient.

Toxicity. The treatment-related AEs are shown in Table II. Grade 3 or 4 AEs were observed in 3 of the 5 patients. The most commonly encountered grade 3 AEs were thrombocytopenia, hypoalbuminemia and hypertension; Grade 4 hyponatremia was observed in 1 patient. None of the patients required discontinuation of this trial treatment due to the emergence of AEs and none of the patients experienced DLTs. There was no case of treatment-related death.

Efficacy. Partial response (PR) was achieved in 1 patient with a maximum tumor size reduction of $67 \%$ (Figure 1). However, this patient died of disease progression 19.9 months after the start of the treatment, with a PFS of 7.4 months. The tumor response was classified as stable disease (SD) in 3 patients and as progressive disease (PD) in 1 patient. All patients eventually died. OS ranged from 2.0 to 19.9 months and PFS from 1.5 to 7.4 months (Table III).

\section{Discussion}

This single-center study was conducted to preliminary evaluate the feasibility and efficacy of axitinib treatment for GEM-refractory BTC. The first clinical suggestion was that axitinib at a starting dose of $5 \mathrm{mg}$ orally twice daily is feasible for GEM-refractory BTC because none of the 5 patients developed DLTs. Axitinib has been approved for the treatment of advanced renal cell carcinoma worldwide, including Japan. Japanese patients were enrolled in a randomized global phase III trial and a single-arm phase II trial for advanced renal cell carcinoma. While hypertension, hand-foot syndrome, diarrhea, proteinuria, hoarseness, fatigue and hypothyroidism were observed as common treatment-related AEs in the two trials, an acceptable safety profile of axitinib was demonstrated $(17,18)$. The AEs in the 5 patients in our current study were comparable to those in these aforementioned trials.

The second clinical suggestion was that axitinib might exert promising activity against GEM-refractory BTC. 
Table I. Patients' characteristics.

\begin{tabular}{lc}
\hline & $\mathrm{n}=5$ \\
\hline Age, years & \\
$\quad$ Median (range) & $68(34-77)$ \\
Gender & $3 / 2$ \\
$\quad$ Male/Female & \\
ECOG performance status & $4 / 1$ \\
0/1 & 2 \\
Primary site & 2 \\
$\quad$ Intrahepatic bile duct & 1 \\
Extrahepatic bile duct & \\
Gallbladder & $3 / 2$ \\
Extent of disease & \\
$\quad$ Metastatic/Recurrent & $2 / 3$ \\
Biliary stent & \\
$\quad$ Yes/No & \\
Prior chemotherapy & 3 \\
First-line chemotherapy & 1 \\
GEM+cisplatin & 1 \\
GEM+S-1 & \\
GEM & 2 \\
Second-line chemotherapy & 1 \\
S-1 & \\
GEM+cisplatin &
\end{tabular}

ECOG, Eastern cooperative oncology group; GEM, gemcitabine; S-1, tegafur, gimeracil and oteracil potassium.

Surgical samples of ECC were investigated for VEGF expression by immunohistochemistry; VEGF was demonstrated as an independent negative prognostic factor by multivariate Cox regression analysis (14). Epidermal growth factor receptor, human epidermal growth factor receptor 2 and VEGF expressions were examined in surgical samples of cholangiocarcinoma (IHCC and ECC) by immunohistochemistry; VEGF overexpression rates were found to be $53.8 \%$ in IHCC and 59.2\% in ECC. Furthermore, VEGF overexpression was associated with intrahepatic metastasis in cases of IHCC (15). Therefore, VEGFR expression is associated with progression of BTC and, thus, attracted attention as a target of treatment in cases of BTC.

Axitinib is an orally administered selective inhibitor of VEGFR-1/2/3 and a highly specific tyrosine kinase inhibitor (TKI) of VEGFR as compared to other known VEGFR TKIs (18). Although clinical trials of multi-TKIs, such as sorafenib and sunitinib, have been conducted in the second-line setting for advanced BTC, none has demonstrated any promising activities against BTC $(4,8)$. A preclinical examination using xenograft models of cholangiocarcinoma showed that axitinib exerted promising anti-tumor efficacy against both GEM-sensitive ECC and GEM-resistant IHCC cell lines (16). Therefore, we conducted this study to preliminary evaluate the efficacy of axitinib in patients with GEM-
Table II. Treatment-related adverse events $(n=5)$.

\begin{tabular}{|c|c|c|}
\hline & Grade $1 / 2$ & Grade 3/4 \\
\hline \multicolumn{3}{|l|}{ Hematological } \\
\hline Thrombocytopenia & 2 & 1 (Grade 3$)$ \\
\hline Neutropenia & 1 & 0 \\
\hline Leukopenia & 1 & 0 \\
\hline Anemia & 1 & 0 \\
\hline \multicolumn{3}{|l|}{ Non-hematological } \\
\hline Malaise & 5 & 0 \\
\hline Fatigue & 4 & 0 \\
\hline Hypertension & 3 & 1 (Grade 3) \\
\hline Proteinuria & 4 & 0 \\
\hline Anorexia & 3 & 0 \\
\hline Diarrhea & 3 & 0 \\
\hline Oral mucositis & 2 & 0 \\
\hline Vomiting & 2 & 0 \\
\hline Hoarseness & 2 & 0 \\
\hline Nausea & 1 & 0 \\
\hline Constipation & 1 & 0 \\
\hline Fever & 1 & 0 \\
\hline Rash & 1 & 0 \\
\hline Arthralgia & 1 & 0 \\
\hline Muscle weakness & 1 & 0 \\
\hline Hematuria & 1 & 0 \\
\hline Hypoalbuminemia & 3 & 1 (Grade 3$)$ \\
\hline TSH (increased) & 4 & 0 \\
\hline Hyponatremia & 1 & 1 (Grade 4$)$ \\
\hline Hypocalcemia & 1 & 0 \\
\hline ALP (increased) & 1 & 0 \\
\hline AST (increased) & 1 & 0 \\
\hline ALT (increased) & 1 & 0 \\
\hline TSH (decreased) & 1 & 0 \\
\hline Blood sugar increased & 1 & 0 \\
\hline
\end{tabular}

TSH, Thyroid stimulating hormone; ALP, alkaline phosphatase; AST, aspartate aminotransferase; ALT, alanine aminotransferase.

refractory BTC. The results revealed a good response $(67 \%$ tumor size regression) in 1 patient, despite the treatment having been administered in the third-line setting (GEM plus cisplatin and S-1 refractory) in this patient.

This study had certain limitations. First, only 5 patients were enrolled in this study. The primary purpose of the study was to confirm feasibility of axitinib for treating GEMrefractory BTC patients; thus, a sample size of 3 to 6 patients was planned. As the next step, a further clinical trial, including a larger sample size, is required to confirm efficacy. Second, biomarker analysis, such as measurement of blood levels of VEGF, VEGFR and soluble VEGFR and tissue expression levels of VEGF and VEGFR, was not conducted in this study. Although axitinib was found to exert promising activity in one patient who showed PR, the relationship between the efficacy and these biomarkers could not be determined. Biomarker analysis is also needed in future studies for precisely evaluating efficacy of axitinib. 
A

B

C
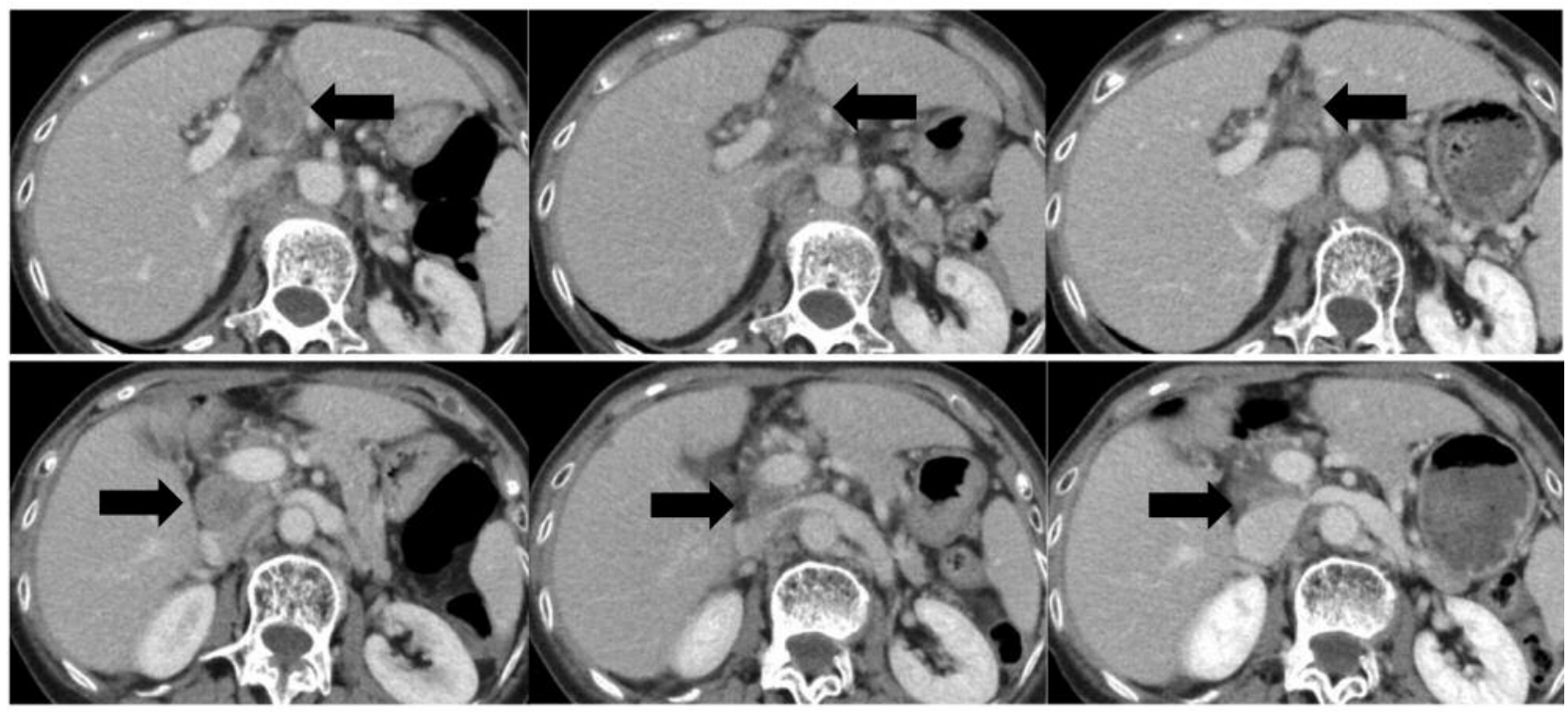

Figure 1. A partial response case. A: Patient with two target lesions in lymph node $(\rightarrow)$ before treatment. B: The target lesions were reduced by $58 \%$ in 2 months after initiation of axitinib. C: Partial response was achieved with a maximum tumor size reduction of 67\% in 4 months after start of axitinib.

Table III. Patient summary.

\begin{tabular}{|c|c|c|c|c|c|}
\hline Patient (years, gender) & Primary site & Prior therapy & Best tumor shrinkage & PFS (mo) & OS (mo) \\
\hline 42 , male & ICC & GC & $17 \%$ tumor regression & 2.4 & 5.6 \\
\hline 77 , male & ECC & GEM followed by S-1 & $9 \%$ tumor regression & 2.9 & 3.6 \\
\hline 68 , male & GBC & $\mathrm{GC}$ & $7 \%$ tumor growth & 2.4 & 7.9 \\
\hline 72 , female & ICC & GC followed by $\mathrm{S}-1$ & $67 \%$ tumor regression & 7.4 & 19.9 \\
\hline 34 , female & $\mathrm{ECC}$ & GS followed by GC & Non-target lesions & 1.5 & 2.0 \\
\hline
\end{tabular}

ICC, Intrahepatic cholangiocarcinoma; ECC, extrahepatic cholangiocarcinoma; GBC, gallbladder cancer; GC, gemcitabine plus cisplatin; GEM, gemcitabine; GS, gemcitabine plus S-1; PFS, progression-free survival; OS, overall survival; mo, months.

In conclusion, axitinib administered at the dose of $5 \mathrm{mg}$ twice daily was well-tolerated, with one patient exhibiting a good response, $67 \%$ reduction of the tumor size, suggesting that axitinib might exert promising activity against BTC. A multicenter single-arm phase II study for GEM-refractory BTC with biomarker analysis is currently ongoing (UMIN000023014).

\section{Acknowledgements}

This research was partially supported by the Practical Research for Innovative Cancer Control from the Japan Agency for Medical Research and development, AMED (15Ack0106079h0002) and also by the Supported Program for the Strategic Research Foundation at Private Universities from the Ministry of Education, Culture, Sports, Science and Technology (S1001004) of Japan. Axitinib was kindly provided by Pfizer.

\section{References}

1 Foundation for Promotion of Cancer Research. Cancer Statistics in Japan-2015.

2 Valle J, Wasan H, Palmer DH, Cunningham D, Anthoney A, Maraveyas A, Madhusudan S, Iveson T, Hughes S, Pereira SP, Roughton $\mathrm{M}$ and Bridgewater $\mathrm{J}$ : Cisplatin plus gemcitabine versus gemcitabine for biliary tract cancer. N Engl J Med 362: 1273-1281, 2010.

3 Okusaka T, Nakachi K, Fukutomi A, Mizuno N, Ohkawa S, Funakoshi A, Nagino M, Kondo S, Nagaoka S, Funai J, Koshiji M, Nambu Y, Furuse J, Miyazaki M and Nimura Y: Gemcitabine alone or in combination with cisplatin in patients with biliary tract cancer: A comparative multicentre study in Japan. Br J Cancer 103: 469-474, 2010.

4 Bengala C, Bertolini F, Malavasi N, Boni C, Aitini E, Dealis C, Zironi S, Depenni R, Fontana A, Del Giovane C, Luppi G and 
Conte P: Sorafenib in patients with advanced biliary tract carcinoma: A phase II trial. Br J Cancer 102: 68-72, 2010.

5 Bekaii-Saab T, Phelps MA, Li X, Saji M, Goff L, Kauh JS, O'Neil BH, Balsom S, Balint C, Liersemann R, Vasko VV, Bloomston M, Marsh W, Doyle LA, Ellison G, Grever M, Ringel MD and Villalona-Calero MA: Multi-institutional phase II study of selumetinib in patients with metastatic biliary cancers. J Clin Oncol 29: 2357-2363, 2011.

6 Oh SY, Jeong CY, Hong SC, Kim TH, Ha CY, Kim HJ, Lee GW, Hwang IG, Jang JS, Kwon HC and Kang JH: Phase II study of second line gemcitabine single chemotherapy for biliary tract cancer patients with 5-fluorouracil refractoriness. Invest New Drugs 29: 1066-1072, 2011.

7 Sasaki T, Isayama H, Nakai Y, Mizuno S, Yamamoto K, Yagioka H, Yashima Y, Kawakubo K, Kogure H, Togawa O, Matsubara S, Sasahira N, Hirano K, Tsujino T, Tada M, Omata M and Koike K: Feasibility study of gemcitabine and cisplatin combination chemotherapy for patients with refractory biliary tract cancer. Invest New Drugs 29: 1488-1493, 2011.

8 Yi JH, Thongprasert S, Lee J, Doval DC, Park SH, Park JO, Park YS, Kang WK and Lim HY: A phase II study of sunitinib as a second-line treatment in advanced biliary tract carcinoma: A multicentre, multinational study. Eur J Cancer 48: 196-201, 2012.

9 Moretto R, Raimondo L, De Stefano A, Cella CA, Matano E, De Placido $\mathrm{S}$ and Carlomagno C: FOLFIRI in patients with locally advanced or metastatic pancreatic or biliary tract carcinoma: A monoinstitutional experience. Anticancer Drugs 24: 980-985, 2013.

10 Sasaki T, Isayama H, Nakai Y, Mizuno S, Yamamoto K, Yagioka H, Yashima Y, Kawakubo K, Kogure H, Togawa O, Matsubara S, Ito Y, Sasahira N, Hirano K, Tsujino T, Toda N, Tada M, Omata $\mathrm{M}$ and Koike K: Multicenter phase II study of S-1 monotherapy as second-line chemotherapy for advanced biliary tract cancer refractory to gemcitabine. Invest New Drugs 30: 708-713, 2012.

11 Suzuki E, Ikeda M, Okusaka T, Nakamori S, Ohkawa S, Nagakawa T, Boku N, Yanagimoto H, Sato T and Furuse J: A multicenter phase II study of S-1 for gemcitabine-refractory biliary tract cancer. Cancer Chemother Pharmacol 71: 1141-1146, 2013.

12 Sasaki T, Isayama H, Nakai Y, Takahara N, Satoh Y, Takai D, Kogure H, Yamamoto N, Hirano K, Tada M, Yatomi Y and Koike $\mathrm{K}$ : A pilot study of salvage irinotecan monotherapy for advanced biliary tract cancer. Anticancer Res 33: 2619-2622, 2013.
13 He S, Shen J, Sun X, Liu L and Dong J: A phase II FOLFOX-4 regimen as second-line treatment in advanced biliary tract cancer refractory to gemcitabine/cisplatin. J Chemother 26: 243-247, 2014.

14 Hida Y, Morita T, Fujita M, Miyasaka Y, Horita S, Fujioka Y, Nagashima $\mathrm{K}$ and Katoh $\mathrm{H}$ : Vascular endothelial growth factor expression is an independent negative predictor in extrahepatic biliary tract carcinomas. Anticancer Res 19: 2257-2260, 1999.

15 Yoshikawa D, Ojima H, Iwasaki M, Hiraoka N, Kosuge T, Kasai $\mathrm{S}$, Hirohashi S and Shibata T: Clinicopathological and prognostic significance of EGFR, VEGF, and HER2 expression in cholangiocarcinoma. Br J Cancer 98: 418-425, 2008.

16 Takahashi H, Ojima H, Shimizu H, Furuse J, Furukawa H and Shibata T: Axitinib (AG-013736), an oral specific VEGFR TKI, shows potential therapeutic utility against cholangiocarcinoma. Jpn J Clin Oncol 44: 570-578, 2014.

17 Rini BI, Escudier B, Tomczak P, Kaprin A, Szczylik C, Hutson TE, Michaelson MD, Gorbunova VA, Gore ME, Rusakov IG, Negrier S, Ou YC, Castellano D, Lim HY, Uemura H, Tarazi J, Cella D, Chen C, Rosbrook B, Kim S and Motzer RJ: Comparative effectiveness of axitinib versus sorafenib in advanced renal cell carcinoma (AXIS): A randomised phase 3 trial. Lancet 378: 1931-1939, 2011.

18 Tomita Y, Uemura H, Fujimoto H, Kanayama HO, Shinohara N, Nakazawa H, Imai K, Umeyama Y, Ozono S, Naito S and Akaza $\mathrm{H}$ : Key predictive factors of axitinib (AG-013736)-induced proteinuria and efficacy: A phase II study in Japanese patients with cytokine-refractory metastatic renal cell carcinoma. Eur J Cancer 47: 2592-2602, 2011.

19 Patson B, R BC and Olszanski AJ: Pharmacokinetic evaluation of axitinib. Expert Opin Drug Metab Toxicol 8: 259-270, 2012.

Received April 24, 2017

Revised May 22, 2017

Accepted May 23, 2017 